PROCEEDINGS OF THE

AMERICAN MATHEMATICAL SOCIETY

Volume 129, Number 7, Pages 2019-2029

S 0002-9939(00)05765-8

Article electronically published on November 30, 2000

\title{
QUASICONFORMAL GROUPS WITH SMALL DILATATION I
}

\author{
PETRA BONFERT-TAYLOR AND GAVEN MARTIN
}

(Communicated by Albert Baernstein II)

\begin{abstract}
We study Fuchsian quasiconformal groups with small dilatation. For this class of groups we establish a Jørgensen-type inequality in all dimensions. We show discreteness persists to the limit under algebraic convergence and that such groups are discrete if and only if every two generator subgroup is discrete.
\end{abstract}

\section{INTRODUCTION}

In this paper we consider quasiconformal groups with small dilatation and begin an investigation into their geometric properties. We establish some basic results, namely a Jørgensen-type inequality and algebraic convergence properties. We are also interested in discovering phenomena which do not in general hold for arbitrary quasiconformal groups. Before we discuss such rigidity phenomena we need to introduce some notation and definitions.

The group of all orientation-preserving Möbius transformations in $\overline{\mathbb{R}^{n}}=\mathbb{R}^{n} \cup$ $\{\infty\}$ is denoted by Möb(n); the subgroup of this group that fixes the open unit ball $\mathbb{B}^{n}$ is denoted $\operatorname{Möb}\left(\mathbb{B}^{n}\right)$. All maps in this article are assumed to be orientationpreserving. A group $G$ of homeomorphisms of $\overline{\mathbb{R}^{n}}$ (or $\overline{\mathbb{B}^{n}}$ ) is discrete if no sequence $\left\{f_{k}\right\} \subset G$ of distinct elements converges to the identity uniformly in $\overline{\mathbb{R}^{n}}$ (or $\overline{\mathbb{B}^{n}}$ ). A discrete subgroup $G$ of Möb(n) is called a Kleinian group (note that some authors require $G$ in addition to be nonelementary).

A group $G$ of homeomorphisms of $\overline{\mathbb{R}^{n}}$ (or $\overline{\mathbb{B}^{n}}$ ) is a $K$-quasiconformal group, $1 \leq K<\infty$, if each of its elements is $K$-quasiconformal, and we call the group simply quasiconformal if it is $K$-quasiconformal for some $K$. The number $K$ is the dilatation of the group. We refer the reader to GeMa for the basic properties of quasiconformal groups. Recall that every Möbius transformation is 1quasiconformal, and conversely every 1-quasiconformal homeomorphism of $\overline{\mathbb{R}^{n}}$ is a Möbius transformation (the Liouville Theorem Geh]).

In this paper we will be mainly interested in quasiconformal groups acting on the unit ball $\mathbb{B}^{n}$. Note that such a group can be uniquely extended to a quasiconformal group acting on $\overline{\mathbb{B}^{n}}$, and can be extended (by reflection, for example) to

Received by the editors April 16, 1999 and, in revised form, November 9, 1999.

1991 Mathematics Subject Classification. Primary 30F40, 57S30, 30C65, 20H10.

The first author acknowledges research support in part by a University of Michigan Rackham fellowship.

The second author acknowledges research support in part by a grant from the NZ Marsden Fund.

(C)2000 American Mathematical Society 
a quasiconformal group acting on $\overline{\mathbb{R}^{n}}$ with the same dilatation; we sometimes call these groups Fuchsian quasiconformal groups.

Sullivan and Gromov showed that every quasiconformal group acts conformally in some bounded measurable Riemannian structure (depending on the group) (see Tuk2, Mar3]). Clearly the quasiconformal conjugate of a Möbius group is a quasiconformal group; the invariant structure is the pull back of the usual structure via the conjugating map. The "measurable Riemann mapping theorem" (the existence theorem for quasiconformal mappings) asserts that in two dimensions any bounded measurable conformal structure is the pullback of the usual structure under a quasiconformal homeomorphism. Thus in dimension 2 every quasiconformal group is a conjugate of a Möbius group ( $\underline{\mathrm{Sul}}]$, Tuk1]). In higher dimensions related arguments show that if the quasiconformal group acts sufficiently ergodically (e.g. conical limit points have full measure), then the invariant structure can be linearized to obtain a quasiconformal conjugacy to a Möbius group.

However in higher dimensions there do exist quasiconformal groups which are not quasiconformally conjugate to Möbius groups (Tuk2], Mar1]). These examples were generalized by McKemie $\underline{\mathrm{McK}}$ who showed that there are such groups with arbitrarily small dilatation. Further, Freedman and Skora gave examples of quasiconformal groups not topologically conjugate to Möbius groups [FrSk]. However such examples appear to need large dilatation and no such examples of small dilatation are known. We ask if all groups with small dilatation are topologically conjugate to Möbius groups. Here and in a sequel we consider the restricted problem of the existence of an isomorphic Möbius group. Freedman was interested in such examples because of his reduction of the 4-dimensional surgery conjecture to the question of lifting certain actions on $\overline{\mathbb{R}^{n}}$ to $\mathbb{R}^{n+1}$. An important open question is whether every quasiconformal group can be so lifted.

In a different direction, in the last few years there have been a number of attempts to generalize some classical results in the theory of Kleinian groups, such as Jørgensen's inequality and other universal constraints (many centering around the Margulis lemma) to higher dimensions and to different types of groups. See for instance Mar2, Mar4, CaWa, Wat, Her, [FrHe]. In each of these generalizations there is (roughly) an underlying invariant metric of negative curvature, and clearly some restriction is necessary. However the first author showed that Jørgensen's inequality (actually its geometric equivalent) does not hold for convergence groups in two dimensions $([\mathrm{BT}])$. Convergence groups have been found to be natural topological analogues of Kleinian groups (see GeMa) and there is now a significant literature concerning them. Quasiconformal groups lie between Möbius groups and convergence groups. It is therefore of interest to decide to what extent geometric constraints, which hold for Möbius groups and fail for convergence groups, hold for quasiconformal groups. Roughly one might say that for small dilatation we are closer to Möbius groups, while for large dilatation we are closer to convergence groups.

There exists a classification of the elements of a quasiconformal group that is topologically analogous to the classification of Möbius maps. If $G$ is a quasiconformal group and $g \in G$, then we say that $g$ is elliptic if $\langle g\rangle$, the group generated by $g$, is precompact. If $g$ is not elliptic, then $g$ is loxodromic if it has exactly two fixed points, or $g$ is parabolic if $g$ fixes exactly one point. It is not hard to see that every element in a quasiconformal group is either elliptic, parabolic or loxodromic. In a discrete convergence group the elliptic elements are those $g \in G$ of finite order. 
The sequence $\left\{g^{k}\right\}$ of iterates of a loxodromic element of a quasiconformal group converges to a fixed point $a$ of $g$ locally uniformly in the exterior of the other fixed point $b$; we call $a$ the attracting and $b$ the repelling fixed point of $g$. For parabolic $g$, the sequence of iterates converges to the fixed point of $g$ locally uniformly in the exterior of that fixed point (see [GeMa]). As with Möbius groups, we define the ordinary set $\Omega(G)$ to be the set of points where $G$ acts discontinuously; i.e. the set of all $x$ that have a neighborhood $U$ satisfying $g(U) \cap U=\varnothing$ for all but finitely many $g \in G$. The limit set $L(G)$ is the complement of the ordinary set. It is roughly the set of limit points (maps) of sequences in $G$. The ordinary set is an open set, and the limit set is closed; both sets are $G$-invariant. If $L(G)$ contains more than two points, then $L(G)$ is an infinite perfect set. If $\Omega(G) \neq \varnothing$, then $G$ is necessarily discrete. See, e.g., GeMa, [Tuk3 for more details here. Note that for a Fuchsian quasiconformal group $G$ we have $L(G) \subset \mathbb{S}^{n-1}$.

As is customary, we define a discrete quasiconformal group $G$ to be nonelementary if the limits set $L(G)$ contains more than two points. We can extend this definition to nondiscrete $G$ but then in addition we must require that no $x \in L(G)$ is fixed by the entire group $G$. In both cases one can show that a quasiconformal group $G$ is elementary if and only if either $L(G)=\varnothing$ or there is a one- or two-point set which is fixed setwise by $G$. Furthermore, $G$ is nonelementary if and only if there are two loxodromic $g, h \in G$ without common fixed points. Nonelementary groups contain free subgroups of arbitrary rank.

The first author wishes to thank Juha Heinonen and Edward Taylor for helpful conversations on this subject.

\section{Distance to the identity}

In this section we shall investigate the distance of a Möbius transformation from the identity.

For a homeomorphism $f: \overline{\mathbb{B}^{n}} \rightarrow \overline{\mathbb{B}^{n}}$ let

$$
d(f, i d):=\max _{x \in \overline{\mathbb{B}^{n}}}|x-f(x)|
$$

denote the maximal Euclidean distance that $f$ moves a point $x \in \overline{\mathbb{B}^{n}}$.

If $f$ is a Möbius transformation, then there is another method to measure the distance of $f$ from the identity: If $A$ is the corresponding matrix in the hyperboloid model (see below), then we can compare the distance of $A$ to the identity matrix with $d(f$,id) (see Lemma 2.1 and Lemma 2.2). The hyperboloid model $2(n)$ is defined by

$$
\mathcal{Q}(n)=\left\{\left(x_{0}, \ldots, x_{n}\right) \in \mathbb{R}^{n+1} \mid q(x, x)=1, x_{0}>0\right\},
$$

where $q(x, y)=x_{0} y_{0}-\left(x_{1} y_{1}+\cdots+x_{n} y_{n}\right)$. Then the map $F: \mathcal{Q}(n) \rightarrow \mathbb{B}^{n}$ given by

$$
F:\left(x_{0}, \ldots, x_{n}\right) \mapsto \frac{1}{1+x_{0}}\left(x_{1}, \ldots, x_{n}\right)
$$

is an isometry between $\mathcal{Q}(n)$ with the metric induced by $q$ (and line element $d s^{2}=$ $\left.d x_{1}^{2}+\cdots+d x_{n}^{2}-d x_{0}^{2}\right)$ and $\mathbb{B}^{n}$ with the hyperbolic metric. The isometries of $\mathcal{Q}(n)$ are the $(n+1) \times(n+1)$-matrices which preserve $q$ and the upper half space $\left\{x \in \mathbb{R}^{n+1} \mid x_{0}>0\right\}$. The group of all such isometries is the rank one Lie subgroup $\mathcal{O}^{+}(1, n)$ of $G L(n+1, \mathbb{R})$. For $f \in \operatorname{Möb}\left(\mathbb{B}^{n}\right)$ the map $A:=F^{-1} \circ f \circ F$ is an isometry of $\mathcal{Q}(n)$ and thus belongs to $\mathcal{O}^{+}(1, n)$. We will refer to $A$ as the matrix 
corresponding to the Möbius transformation $f$. In terms of the matrix $A=\left(a_{i j}\right)$, for $x \in \mathbb{B}^{n}$ the image $y=f(x)$ has coordinates

$$
y_{j}=\frac{\left(1+|x|^{2}\right) a_{j 0}+2\left(x_{1} a_{j 1}+\cdots+x_{n} a_{j n}\right)}{|x|^{2}\left(a_{00}-1\right)+2\left(x_{1} a_{01}+\cdots+x_{n} a_{0 n}\right)+\left(a_{00}+1\right)} .
$$

For a detailed exposition see [Bea, pp. 48-54].

Let now $f \in \operatorname{Möb}\left(\mathbb{B}^{n}\right)$ and let $A \in \operatorname{Isom}(Q(n))$ be the corresponding matrix in the hyperboloid model. Let furthermore

$$
\|A-\mathrm{I}\|_{\infty}:=\sup _{x \in \mathbb{R}^{n+1}, x \neq 0} \frac{|A x-x|}{|x|}=\max _{|x|=1}|A x-x|
$$

be the distance of the matrix $A$ from the identity matrix, measured in the operator norm, and let

$$
\|A-\mathrm{I}\|:=\sum_{i \neq j} a_{i j}^{2}+\sum_{i=1}^{n+1}\left(a_{i i}-1\right)^{2}
$$

denote the distance of $A$ from the identity matrix, measured in the Hilbert-Schmidt norm.

We now give some inequalities relating the distances $d(f, \mathrm{id}),\|A-\mathrm{I}\|_{\infty}$, and $\|A-\mathrm{I}\|$.

Lemma 2.1. If $f \in \operatorname{Möb}\left(\mathbb{B}^{n}\right)$ and $A \in \operatorname{Isom}(Q(n))$ is the corresponding matrix in the hyperboloid model, then

$$
d(f, \text { id }) \leq \sqrt{8}\|A-\mathrm{I}\|_{\infty} \leq \sqrt{8}\|A-\mathrm{I}\| .
$$

For an inequality in the other direction we have:

Lemma 2.2. If $f \in \operatorname{Möb}\left(\mathbb{B}^{n}\right)$ and $A \in \operatorname{Isom}(Q(n))$ is the corresponding matrix in the hyperboloid model, then

$$
\|A-\mathrm{I}\|<\sqrt{16+n} \frac{d(f, \mathrm{id})}{1-d(f, \mathrm{id})^{2}}
$$

whenever $d(f, \mathrm{id})<1$.

Proof of Lemma 2.1] Let $\widetilde{x}, \widetilde{y} \in \mathcal{Q}(n), \widetilde{x}=\left(x_{0}, \ldots, x_{n}\right), \widetilde{y}=\left(y_{0}, \ldots, y_{n}\right)$, and $x:=F(\widetilde{x}), y:=F(\widetilde{y})$ be the corresponding points in $\mathbb{B}^{n}$. We shall show that

$$
|x-y| \leq \sqrt{8} \cdot \frac{|\widetilde{x}-\widetilde{y}|}{|\widetilde{x}|} .
$$

Choosing $\widetilde{y}=A \widetilde{x}$, this proves $|x-f(x)| \leq \sqrt{8}|A \widetilde{x}-\widetilde{x}| /|\widetilde{x}| \leq \sqrt{8}|| A-\mathrm{I} \|_{\infty}$ and thus $d(f$, id $)=\sup _{x \in \mathbb{B}^{n}}|x-f(x)| \leq \sqrt{8}|| A-\mathrm{I} \|_{\infty}$.

Hence we only have to prove (11). To this end we may assume without loss of generality that $\widetilde{x}$ has the special form $\widetilde{x}=\left(x_{0}, \sqrt{x_{0}^{2}-1}, 0, \ldots, 0\right)$, and hence $x=\left(\frac{\sqrt{x_{0}^{2}-1}}{x_{0}+1}, 0, \ldots, 0\right)$. Then

$$
\begin{aligned}
|\widetilde{x}-\widetilde{y}|^{2} & =\left(x_{0}-y_{0}\right)^{2}+x_{0}^{2}-1-2 \sqrt{x_{0}^{2}-1} y_{1}+y_{0}^{2}-1, \\
|x-y|^{2} & =\frac{2 x_{0} y_{0}-2 \sqrt{x_{0}^{2}-1} y_{1}-2}{\left(x_{0}+1\right)\left(y_{0}+1\right)}, \text { and } \\
|\widetilde{x}|^{2} & =2 x_{0}^{2}-1,
\end{aligned}
$$


and hence
(2) $\frac{|x-y|^{2}}{\frac{|\widetilde{x}-\tilde{x}|^{2}}{|\widetilde{x}|^{2}}}=\frac{x_{0} y_{0}-\sqrt{x_{0}^{2}-1} y_{1}-1}{x_{0}^{2}+y_{0}^{2}-x_{0} y_{0}-\sqrt{x_{0}^{2}-1} y_{1}-1} \cdot \frac{2 x_{0}^{2}-1}{\left(x_{0}+1\right)\left(y_{0}+1\right)}$

$$
=\left(1-\frac{\left(x_{0}-y_{0}\right)^{2}}{\left(x_{0}-y_{0}\right)^{2}+x_{0} y_{0}-\sqrt{x_{0}^{2}-1} y_{1}-1}\right) \cdot \frac{2 x_{0}^{2}-1}{\left(x_{0}+1\right)\left(y_{0}+1\right)} .
$$

Observe that since $\widetilde{y} \in \mathcal{Q}(n)$ we have

$$
\begin{aligned}
y_{0} & \geq 1 \text { and } \\
-\sqrt{y_{0}^{2}-1} \leq y_{1} & \leq \sqrt{y_{0}^{2}-1}
\end{aligned}
$$

Since $\frac{|x-y|^{2}}{\frac{|\widetilde{x}-\widetilde{y}|^{2}}{|\widetilde{x}|^{2}}} \geq 0$ and $|\widetilde{x}-\widetilde{y}| \geq 0$, we obtain from (3) that

$$
0 \leq \frac{\left(x_{0}-y_{0}\right)^{2}}{\left(x_{0}-y_{0}\right)^{2}+x_{0} y_{0}-\sqrt{x_{0}^{2}-1} y_{1}-1} \leq 1
$$

We distinguish three cases.

(i) If $x_{0} \leq y_{0}$, then (3) and (6) yield

$$
\frac{|x-y|^{2}}{\frac{|\tilde{x}-\tilde{y}|^{2}}{|\widetilde{x}|^{2}}} \leq 1 \cdot \frac{2 x_{0}^{2}-1}{\left(x_{0}+1\right)^{2}} \leq 2 .
$$

(ii) If $x_{0}>y_{0}$ and $x_{0} \leq 2$, then (3i) and (6) yield

$$
\frac{|x-y|^{2}}{\frac{|\widetilde{x}-\widetilde{y}|^{2}}{|\widetilde{x}|^{2}}} \leq 1 \cdot \frac{2 x_{0}^{2}-1}{\left(x_{0}+1\right) \cdot 2}=x_{0}-\frac{2 x_{0}+1}{2 x_{0}+2} \leq x_{0} \leq 2 .
$$

(iii) If $x_{0}>y_{0}$ and $x_{0}>2$, then (2) and (5) yield

$$
\begin{aligned}
\frac{|x-y|^{2}}{\frac{|\widetilde{x}-\tilde{y}|^{2}}{|\widetilde{x}|^{2}}} & \leq \frac{x_{0} y_{0}+\sqrt{x_{0}^{2}-1} \sqrt{y_{0}^{2}-1}-1}{\left(x_{0}+1\right)\left(y_{0}+1\right)} \cdot \frac{2 x_{0}^{2}-1}{x_{0}^{2}+y_{0}^{2}-x_{0} y_{0}+\sqrt{x_{0}^{2}-1} \sqrt{y_{0}^{2}-1}-1} \\
& \leq \frac{2 x_{0} y_{0}}{x_{0} y_{0}} \cdot \frac{2 x_{0}^{2}}{x_{0}^{2}+y_{0}^{2}-x_{0} y_{0}+\sqrt{x_{0}^{2}-1} \sqrt{y_{0}^{2}-1}-1} .
\end{aligned}
$$

Consider (for fixed $x_{0} \geq 2$ and $1 \leq y_{0}<x_{0}$ ) the function

$$
h\left(y_{0}\right):=\frac{2 x_{0}^{2}}{x_{0}^{2}+y_{0}^{2}-x_{0} y_{0}+\sqrt{x_{0}^{2}-1} \sqrt{y_{0}^{2}-1}-1} .
$$

We have $h(1)=2+\frac{2}{x_{0}-1} \leq 4$ and

$$
\begin{aligned}
h^{\prime}\left(y_{0}\right) & =\frac{-2 x_{0}^{2}\left(x_{0}-y_{0}\right)\left(2 y_{0}^{2}-1+x_{0} y_{0}-\sqrt{y_{0}^{2}-1} \sqrt{x_{0}^{2}-1}\right)}{\sqrt{y_{0}^{2}-1}\left(\sqrt{x_{0}^{2}-1}-\sqrt{y_{0}^{2}-1}\right)\left(x_{0}^{2}+y_{0}^{2}-x_{0} y_{0}+\sqrt{x_{0}^{2}-1} \sqrt{y_{0}^{2}-1}-1\right)^{2}} \\
& <0 .
\end{aligned}
$$

Hence $h\left(y_{0}\right) \leq 4$ for all $1 \leq y_{0}<x_{0}$ and thus $|\widetilde{x}|^{2}|x-y|^{2} /|\widetilde{x}-\widetilde{y}|^{2} \leq 8$.

This proves (11). The second inequality in Lemma 2.1 is just an inequality between different matrix norms and is well known, so that the proof of the Lemma is complete. 
Proof of Lemma [2.2, This inequality is a more precise version of [Mar2, Thm. 2.9]; it is essentially contained in the proof of the mentioned theorem. We do not repeat the details here.

\section{The Commutator}

In this section we compare the distance of the commutator of two maps from the identity with their individual distances from the identity. For two homeomorphisms $f, g: \overline{\mathbb{B}^{n}} \rightarrow \overline{\mathbb{B}^{n}}$ let

$$
[f, g]:=f \circ g \circ f^{-1} \circ g^{-1}: \overline{\mathbb{B}^{n}} \rightarrow \overline{\mathbb{B}^{n}}
$$

denote their commutator. Similarly, for two matrices $A, B$ let $[A, B]:=A B A^{-1} B^{-1}$.

The basis of our proof of a form of Jørgensen's inequality rests on the following:

Theorem 3.1. For each $n \geq 2$ there exist two constants $d_{n}>0$ and $K_{0}(n)>1$ that only depend on $n$ such that all $K$-quasiconformal maps $f, g: \overline{\mathbb{B}^{n}} \rightarrow \overline{\mathbb{B}^{n}}$ with $K \leq K_{0}(n)$ satisfy the following: If $d(f, \mathrm{id}) \leq d_{n}$ and $d(g$,id $) \leq d_{n}$, then

$$
d([f, g], \text { id }) \leq \frac{3}{4} \min \{d(f, \text { id }), d(g, \text { id })\} .
$$

In order to show this theorem we first prove the corresponding statement in the Möbius case. The theorem will then follow by a limiting argument.

Lemma 3.2. For each $n \geq 2$ there exists a constant $d_{n}>0$ such that all $f, g \in$ $\operatorname{Möb}\left(\mathbb{B}^{n}\right)$ satisfy the following: If $d(f, \mathrm{id}) \leq d_{n}$ and $d(g, \mathrm{id}) \leq d_{n}$, then

$$
d([f, g], \text { id }) \leq \frac{1}{2} \min \{d(f, \text { id }), d(g, \text { id })\} .
$$

One may choose $d_{n}=\frac{9}{32} \frac{(\sqrt{3}-1)^{2}}{\sqrt{8}(16+n)}$.

Proof of Lemma 3.2. We will use the following result [Mar2, (3.2)]: If $A, B$ are the matrix representations in $Q(n)$ of the Möbius maps $f, g$, then

$$
\|[A, B]-\mathrm{I}\| \leq \frac{r}{(1-r)^{2}} \min (\|A-\mathrm{I}\|,\|B-\mathrm{I}\|)
$$

whenever $\|A-\mathrm{I}\|<r,\|B-\mathrm{I}\|<r$, and $r<2-\sqrt{3}$.

Let now $r<2-\sqrt{3}$ and $f, g \in \operatorname{Möb}\left(\mathbb{B}^{n}\right)$ with $d(f$,id $) \leq \frac{3 r}{4 \sqrt{16+n}}$ and $d(g$,id $) \leq$ $\frac{3 r}{4 \sqrt{16+n}}$. Let $A, B$ be the corresponding matrices in $\mathcal{Q}(n)$. Using Lemma 2.2 and the fact that $d(f$, id $) \leq \frac{1}{2}$ we obtain

$$
\|A-\mathrm{I}\| \leq \sqrt{16+n} \frac{d(f, \mathrm{id})}{1-d(f, \mathrm{id})^{2}} \leq \frac{4}{3} \sqrt{16+n} d(f, \mathrm{id}) \leq r .
$$

Similarly $\|B-\mathrm{I}\| \leq r$. Hence we can apply (7) and obtain, using Lemma 2.1 (7), and Lemma 2.2

$$
d([f, g], \mathrm{id}) \leq \frac{\sqrt{8} r \sqrt{16+n}}{(1-r)^{2}} \min \left\{\frac{d(f, \mathrm{id})}{1-d(f, \mathrm{id})^{2}}, \frac{d(g, \mathrm{id})}{1-d(g, \mathrm{id})^{2}}\right\} .
$$

Now $d(f$, id $) \leq \frac{1}{2}, d(g$, id $) \leq \frac{1}{2}$ and $r \leq 2-\sqrt{3}$ yield

$$
d([f, g], \text { id }) \leq \frac{4 r \sqrt{8} \sqrt{16+n}}{3(\sqrt{3}-1)^{2}} \min \{d(f, \text { id }), d(g, \text { id })\} .
$$


Choose $r=\frac{1}{2} \cdot \frac{3(\sqrt{3}-1)^{2}}{4 \sqrt{8} \sqrt{16+n}}$. Then $r<2-\sqrt{3}$, and

$$
d([f, g], \mathrm{id}) \leq \frac{1}{2} \min \{d(f, i d), d(g, \mathrm{id})\}
$$

and this proves the lemma.

The proof of Theorem 3.1 can now be deduced from Lemma 3.2 by a limiting argument.

Proof of Theorem 3.1. Choose $d_{n}$ as in Lemma 3.2. Suppose the statement of the theorem were not true, i.e. the constant $K_{0}(n)>1$ does not exist. Then there exist $K_{j}$-quasiconformal maps $f_{j}, g_{j}: \overline{\mathbb{B}^{n}} \rightarrow \overline{\mathbb{B}^{n}}$ where $K_{j} \rightarrow 1$ as $j \rightarrow \infty$, so that $d\left(f_{j}, \mathrm{id}\right) \leq d_{n}$ and $d\left(g_{j}, \mathrm{id}\right) \leq d_{n}$ and

$$
d\left(\left[f_{j}, g_{j}\right], \mathrm{id}\right)>\frac{3}{4} \min \left\{d\left(f_{j}, \mathrm{id}\right), d\left(g_{j}, \mathrm{id}\right)\right\}
$$

Passing to a subsequence we may assume that $\left\{f_{j}\right\}$ converges to a Möbius transformation $f$ and $\left\{g_{j}\right\}$ converges to a Möbius transformation $g$ as $j \rightarrow \infty$ (see Vai. Thm. 20.5, Cor. 21.3, Cor. 37.3]); we have made use of continuity of $d(\cdot, \mathrm{id})$ and the fact that $d\left(f_{j}\right.$,id $) \leq d_{n}<2$ and $d\left(g_{j}\right.$, id $) \leq d_{n}$ to eliminate the possibility that the limit map might be constant. Again by continuity of $d(\cdot$, id $)$ we obtain for large enough $j$ that

$$
\begin{aligned}
d\left(\left[f_{j}, g_{j}\right], \mathrm{id}\right) & \leq \sqrt{\frac{3}{2}} d([f, g], \mathrm{id}) \\
& \leq \frac{1}{2} \sqrt{\frac{3}{2}} \min \{d(f, \mathrm{id}), d(g, \mathrm{id})\} \quad \text { by Lemma 3.2 } \\
& \leq \frac{1}{2} \frac{3}{2} \min \left\{d\left(f_{j}, \mathrm{id}\right), d\left(g_{j}, \mathrm{id}\right)\right\},
\end{aligned}
$$

and this contradicts (8)

\section{JøRGENSEN'S INEQUALITY}

Theorem 3.1 now enables us to prove a Jørgensen-type inequality for quasiconformal groups with small dilatation, acting on $\overline{\mathbb{B}^{n}}$ (Theorem 4.2). For Kleinian groups an important consequence of Jørgensen's inequality is roughly that the space of nonelementary Kleinian groups is closed; that is, discreteness is preserved in limits. We will generalize this result to quasiconformal groups with small dilatation (Theorem 4.4).

First we show that limits of quasiconformal groups are quasiconformal groups (without regard to discreteness).

Lemma 4.1. Let $G$ be an abstract group and let $\varphi_{i}: G \rightarrow G_{i}, i=0,1, \ldots$, be homomorphisms of $G$ onto $K$-quasiconformal groups on $\overline{\mathbb{B}^{n}}$, not necessarily discrete. Suppose that for each $g \in G$ we have an open set $U_{g} \subset \overline{\mathbb{B}^{n}}$ such that

$$
\varphi_{i}(g) \rightarrow \varphi_{\infty}(g) \quad \text { locally uniformly in } U_{g},
$$

where $\varphi_{\infty}(g)$ is a nonconstant mapping. Then $G_{\infty}:=\left\{\varphi_{\infty}(g) \mid g \in G\right\}$ is a Kquasiconformal group acting on $\overline{\mathbb{B}^{n}}$.

Furthermore, if $G$ is finitely generated, then it suffices to assume (9) for the generators $g_{1}, \ldots, g_{m}$ of $G$; in this case $G_{\infty}=\left\langle\varphi_{\infty}\left(g_{1}\right), \ldots, \varphi_{\infty}\left(g_{m}\right)\right\rangle$ and it again follows that $G_{\infty}$ is a $K$-quasiconformal group. 
We will see later (Theorem 4.4) that under the additional assumptions that $G$ is torsion-free, all $\varphi_{i}$ are isomorphisms, and all $G_{i}$ are discrete and nonelementary quasiconformal groups with small dilatation, we can deduce that $\varphi_{\infty}$ is an isomorphism as well and $G_{\infty}$ is a discrete, nonelementary quasiconformal group.

Proof of Lemma 4.1. The convergence properties of sequences of quasiconformal mappings show that the hypotheses imply $\varphi_{i}(g) \rightarrow \varphi_{\infty}(g)$ uniformly in $\overline{\mathbb{B}^{n}}$ for each $g \in G$ (for each generator $g_{j}$ in the finitely generated case), and that each $\varphi_{\infty}(g)$ $\left(\varphi_{\infty}\left(g_{j}\right)\right.$ respectively) is a $K$-quasiconformal homeomorphism.

In the finitely generated case we obtain that $G_{\infty}$ is a group of quasiconformal maps generated by $K$-quasiconformal maps. We need to show that the dilatation of each element of $G_{\infty}$ is bounded above by $K$ as well. Let $h \in G_{\infty}$. We write $h$ as a word in our generating set $\varphi_{\infty}\left(g_{1}\right), \ldots, \varphi_{\infty}\left(g_{m}\right)$. The corresponding word in $G_{i}$, written in its generating set $\varphi_{i}\left(g_{1}\right), \ldots, \varphi_{i}\left(g_{m}\right)$, is $h_{i}$. Now $h_{i}$ is $K$-quasiconformal (independently of $i$ ), and the uniform convergence of the generators implies that $\left\{h_{i}\right\}$ converges to $h$ uniformly, which must then be $K$-quasiconformal. This shows that in the finitely generated case, $G_{\infty}$ is a group of $K$-quasiconformal mappings as well.

Finally, since each $\varphi_{i}(g), g \in G, i \in \mathbb{N}$, keeps $\overline{\mathbb{B}^{n}}$ invariant, this property persists in the limit. Thus $G_{\infty}$ is a $K$-quasiconformal group acting on $\overline{\mathbb{B}^{n}}$.

Theorem 4.2 (Jørgensen's inequality). Let $n \geq 2$ and $K \leq K_{0}(n)$ (see Theorem 3.1). Let $f, g$ generate a discrete, nonelementary $K$-quasiconformal group on $\overline{\mathbb{B}^{n}}$, and suppose $f$ is not elliptic. Then

$$
\max \{d(f, \mathrm{id}), d(g, \mathrm{id})\} \geq d_{n},
$$

where $d_{n}$ (which only depends on $n$ ) is the constant from Lemma 3.2.

Proof of Theorem 4.2. Suppose to the contrary that $d(f$, id $)<d_{n}$ and $d(g$, id $)<d_{n}$; we seek a contradiction. Define $g_{0}:=g$ and $g_{j+1}:=\left[g_{j}, f\right]$ for $j \geq 1$. Inductively, using Theorem 3.1 , we obtain that $d\left(g_{j}\right.$, id $)<d_{n}$ for all $j$, and more precisely that

$$
d\left(g_{j}, \mathrm{id}\right) \leq\left(\frac{3}{4}\right)^{j} \min \{d(f, \mathrm{id}), d(g, \mathrm{id})\} .
$$

Since $\langle f, g\rangle$ is discrete by assumption, we conclude that $g_{j_{0}}=$ id for some $j_{0} \in \mathbb{N}$. Define now $h_{0}:=g$ and $h_{j+1}:=h_{j} \circ f \circ h_{j}^{-1}$ for all $j \in \mathbb{N}$. Inductively one sees that $h_{j+1}=g_{j} \circ f \circ g_{j}^{-1}$. Hence $h_{j_{0}}=f$. We distinguish two cases.

(i) Suppose first that $f$ is parabolic. Then $f$ has a unique fixed point $a \in \mathbb{S}^{n-1}$. Since each $h_{j}$ is conjugate to $f$ for $j \geq 1$, each such $h_{j}$ is parabolic and has a unique fixed point; $h_{j+1}$ fixes $h_{j}(a)$ for $j \geq 0$. Thus $h_{j_{0}}$ fixes $h_{j_{0}-1}(a)$, but since $h_{j_{0}}=f$, this implies that $h_{j_{0}-1}(a)=a$, therefore $h_{j_{0}-1}$ fixes $a$. In the same way we obtain that $h_{j_{0}-2}$ fixes $a$, and so on, finally giving that $h_{0}$ fixes $a$, i.e. $g(a)=a$. This shows that $\langle f, g\rangle$ is elementary, contradicting our assumption.

(ii) Suppose next that $f$ is loxodromic. Then $f$ fixes exactly two points $a, b \in$ $\mathbb{S}^{n-1}$. Each $h_{j}$ is conjugate to $f$ for $j \geq 1$ and thus loxodromic, too; $h_{j+1}$ fixes $h_{j}(a)$ and $h_{j}(b)$. Since $h_{j_{0}}=f$ fixes $a, b$, this shows that $\{a, b\}=$ $\left\{h_{j_{0}-1}(a), h_{j_{0}-1}(b)\right\}$, i.e. $h_{j_{0}-1}$ either interchanges or fixes $a$ and $b$. In the first case, $h_{j_{0}-1}^{2}$ fixes $a, b$ and the two loxodromic fixed points of $h_{j_{0}-1}$, which 
contradicts the fact that $h_{j_{0}-1}$ is loxodromic. Thus $h_{j_{0}-1}$ fixes $a, b$, and proceeding in the same way we obtain that $g$ fixes $a$ and $b$. Again, this shows that $\langle f, g\rangle$ is elementary, contradicting our assumption.

We can also formulate Theorem 4.2 in terms of the commutator $[f, g]$.

Corollary 4.3. Let $f, g$ generate a torsion-free, discrete, nonelementary, $K$-quasiconformal group on $\overline{\mathbb{B}^{n}}$, where $K \leq K_{0}(n)$. Then

$$
\begin{aligned}
& \max \{d(f, \text { id }), d([f, g], \text { id })\} \geq d_{n} \text { and } \\
& \max \{d(g, \text { id }), d([f, g], \text { id })\} \geq d_{n} .
\end{aligned}
$$

Proof of Corollary 4.3 Using the fact that $\langle f, g\rangle$ is nonelementary and torsion-free, it is easy to see that $\left\langle f, g \circ f \circ g^{-1}\right\rangle$ is nonelementary as well. Since

$$
\left\langle f, g \circ f \circ g^{-1}\right\rangle=\left\langle f, g \circ f \circ g^{-1} \circ f^{-1}\right\rangle,
$$

we conclude that $\langle f,[f, g]\rangle$ is a torsion-free, discrete, nonelementary, $K$-quasiconformal group on $\mathbb{B}^{n}$. Thus Theorem 4.2 implies that the first inequality of the corollary holds, and the second inequality follows similarly.

As in the Möbius case we can now prove a theorem on algebraic convergence.

Theorem 4.4. Let $G$ be an abstract torsion-free group. Let $\varphi_{i}: G \rightarrow G_{i}, i=$ $0,1 \ldots$, be isomorphisms of $G$ onto discrete, nonelementary, $K_{i}$-quasiconformal groups $G_{i}$ acting on $\overline{\mathbb{B}^{n}}$, where $K_{i} \leq K_{0}(n)$. Suppose furthermore that the sequence $\left\{\varphi_{i}\right\}$ converges algebraically to $\varphi_{\infty}: G \rightarrow G_{\infty}$ (i.e. for each $g \in G$ the limit $\varphi_{\infty}(g):=\lim _{i \rightarrow \infty} \varphi_{i}(g)$ exists and is a homeomorphism of $\overline{\mathbb{B}^{n}}$, and $G_{\infty}:=$ $\left.\left\{\varphi_{\infty}(g) \mid g \in G\right\}\right)$. Then $\varphi_{\infty}$ is an isomorphism and $G_{\infty}$ is a discrete nonelementary $K$-quasiconformal group acting on $\overline{\mathbb{B}^{n}}$.

Proof of Theorem 4.4. From Theorem 4.1, the limit group $G_{\infty}$ is a quasiconformal group acting on $\mathbb{B}^{n}$. Next the algebraic convergence assumption implies $\varphi_{\infty}$ is a homomorphism. Suppose that $\varphi_{\infty}$ is not injective; we seek a contradiction. Set $\psi=\varphi_{\infty} \circ \varphi_{0}^{-1}: G_{0} \rightarrow G_{\infty}$.

If $\psi$ is not injective, then $H:=\operatorname{Ker}(\psi)$ is a nontrivial subgroup of $G_{0}$. We first show that $H$ is nonelementary. Let $f \in H, f \neq$ id. Since $G_{0}$ is nonelementary, there exists $h \in G_{0}$ so that $f$ and $h$ have disjoint fixed point sets. Let $a$ be a fixed point of $f$. Then all $h^{m}(a), m \in \mathbb{N}$, are mutually distinct. Since $H$ is a normal subgroup of $G_{0}$, we have $h^{m} \circ f \circ h^{-m} \in H$ for all $m$. But these elements fix $h^{m}(a)$, so that the limit set of $H$ contains more than two points, and thus $H$ is nonelementary.

Hence there exist two loxodromic elements $f, g \in H$ with disjoint fixed point sets, i.e. $\langle f, g\rangle$ is nonelementary. Then also the fixed point sets of $f_{i}:=\left(\varphi_{i} \circ \varphi_{0}^{-1}\right)(f) \in G_{i}$ and $g_{i}:=\left(\varphi_{i} \circ \varphi_{0}^{-1}\right)(g) \in G_{i}$ are disjoint for each $i$ by [GeMa, Corollary 6.9], the classification of the elementary discrete groups, and the fact that $\varphi_{i}$ is an isomorphism. Hence we can apply Theorem 4.2 to the group $\left\langle f_{i}, g_{i}\right\rangle \subset G_{i}$ and obtain

$$
\max \left\{d\left(f_{i}, \mathrm{id}\right), d\left(g_{i}, \mathrm{id}\right)\right\} \geq d_{n},
$$

contradicting the fact that $f_{i} \rightarrow \psi(f)=$ id and $g_{i} \rightarrow \psi(g)=$ id uniformly on $\overline{\mathbb{R}^{n}}$.

We now need to show that the limit group $G_{\infty}$, which is already known to be a quasiconformal group, is discrete and nonelementary. It is easy to see $G_{\infty}$ is 
nonelementary since $\psi$ is an isomorphism. Suppose that $G_{\infty}$ is not discrete. Then there are $g_{j} \in G_{\infty}$ such that $g_{j} \rightarrow$ id as $j \rightarrow \infty$. Since $G_{\infty}$ is nonelementary, we find $f_{1}$ and $f_{2}$ in $G_{\infty}$ with disjoint fixed point sets. Furthermore, we have $g_{j} \circ f_{k} \circ g_{j}^{-1} \rightarrow$ $f_{k}$ uniformly as $j \rightarrow \infty, k=1,2$, and $\left(\varphi_{i} \circ \varphi_{\infty}^{-1}\right)\left(g_{j} \circ f_{k} \circ g_{j}^{-1}\right) \rightarrow g_{j} \circ f_{k} \circ g_{j}^{-1}$ uniformly as $i \rightarrow \infty$ for each (fixed) $j \in \mathbb{N}$. Hence there is $j_{0} \in \mathbb{N}$ and $i_{0}(j)$ for each $j \geq j_{0}$ such that for $j \geq j_{0}, i \geq i_{0}(j)$ we have

$$
\begin{aligned}
d\left(g_{j} \circ f_{k} \circ g_{j}^{-1}, \mathrm{id}\right) & <\frac{d_{n}}{3} \text { and } \\
d\left(\left(\varphi_{i} \circ \varphi_{\infty}^{-1}\right)\left(g_{j} \circ f_{k} \circ g_{j}^{-1}\right), g_{j} \circ f_{k} \circ g_{j}^{-1}\right) & <\frac{d_{n}}{3} .
\end{aligned}
$$

Using the triangle inequality we conclude from this that

$$
d\left(\left(\varphi_{i} \circ \varphi_{\infty}^{-1}\right)\left(g_{j}\right), \mathrm{id}\right)<d_{n} \text { and } d\left(\left[\left(\varphi_{i} \circ \varphi_{\infty}^{-1}\right)\left(g_{j}\right),\left(\varphi_{i} \circ \varphi_{0}^{-1}\right)\left(f_{k}\right)\right]\right)<d_{n}
$$

Hence, by Corollary 4.3, the group $\left\langle\left(\varphi_{i} \circ \varphi_{\infty}^{-1}\right)\left(g_{j}\right),\left(\varphi_{i} \circ \varphi_{\infty}^{-1}\right)\left(f_{k}\right)\right\rangle$ is elementary for $k=1,2, j \geq j_{0}, i \geq i_{0}(j)$. We conclude that $\left(\varphi_{i} \circ \varphi_{\infty}^{-1}\right)\left(g_{j}\right)$ and $\left(\varphi_{i} \circ \varphi_{\infty}^{-1}\right)\left(f_{k}\right)$ have a fixed point in common for each $i \geq i_{0}(j)$. By [GeMa, Thm. 6.7] the elements $\left(\varphi_{i} \circ \varphi_{\infty}^{-1}\right)\left(g_{j}\right)$ and $\left(\varphi_{i} \circ \varphi_{\infty}^{-1}\right)\left(f_{k}\right)$ are either both loxodromic or both parabolic. Hence, $\left(\varphi_{i} \circ \varphi_{\infty}^{-1}\right)\left(f_{1}\right)$ and $\left(\varphi_{i} \circ \varphi_{\infty}^{-1}\right)\left(f_{2}\right)$ are of the same type and share their fixed points, generating a virtually abelian subgroup. Since $\left(\varphi_{i} \circ \varphi_{\infty}^{-1}\right)$ is an isomorphism $f_{1}$ and $f_{2}$ generate a virtually abelian torsion-free group and therefore have a common fixed point, contradicting our assumption.

Another corollary of Theorem 4.2 is the following:

Corollary 4.5. Let $G$ be a nonelementary, torsion-free $K$-quasiconformal group on $\overline{\mathbb{B}^{n}}$, where $K \leq K_{0}(n)$. Then $G$ is discrete if and only if every subgroup generated by two of its elements is discrete.

The proof of this corollary is a minor modification of Jørgensen's proof in the Möbius case $\mathrm{J \varnothing r}$; we shall not repeat it here.

In a sequel we will discuss further applications of our version of Jørgensen's inequality for quasiconformal groups with small dilatation, including a stability result which shows that under fairly general circumstances a quasiconformal group with small dilatation is isomorphic to a Möbius group.

\section{REFERENCES}

[Bea] A.F. Beardon, The geometry of discrete groups Springer-Verlag, New York, 1983. MR 97d:22011

[BT] P. Bonfert-Taylor, Jørgensen's inequality for discrete convergence groups, Ann. Acad. Sci. Fenn. Math. 25 (2000) 131-150.

[CaWa] C. Cao and P. Waterman, Conjugacy invariants of Möbius groups, Quasiconformal mappings and analysis, Springer-Verlag, 1997. MR 98k:30058

[FrHe] S. Friedland and S. Hersonsky, Jørgensen's inequality for discrete groups in normed algebras, Duke Math. J. 69 (1993) 593-614. MR 94c:46100

[FrSk] M.H. Freedman and R. Skora, Strange actions of groups on spheres, J. Differential Geom. 25 (1987) 75-98. MR 88a:57074

[Geh] F.W. Gehring, Rings and quasiconformal mappings in space, Trans. Amer. Math. Soc. 103 (1962) 353-393. MR 25:3166

[GeMa] F.W. Gehring and G.J. Martin, Discrete quasiconformal groups I, Proc. London Math. Soc. (3) 55 (1987) 331-358. MR 88m:30057

[Her] S. Hersonsky, A generalization of Shimitzu-Leutbecher and Jørgensen's inequalities to Möbius transformation in $\mathbb{R}^{n}$. Proc. Amer. Math. Soc. 121 (1994) 209-215. MR 94m:30085 
[Jør] T. Jørgenson, On discrete groups of Möbius transformations, Amer. J. Math. 96 (1976) 739-749.

[Mar1] G.J. Martin, Discrete quasiconformal groups that are not the quasiconformal conjugates of Möbius groups, Ann. Acad. Sci. Fenn. Ser. AI Math. 11 (1986) 179-202. MR 89d:30025

[Mar2] G.J. Martin, Discrete Möbius groups in all dimensions: A generalization of Jørgensen's inequality, Acta Math. 163 (1989) 253-289. MR 91g:30049

[Mar3] G.J. Martin, Quasiconformal and affine groups. J. Diff. Geom. 29 (1989) 427-448. MR 90a:30064

[Mar4] G.J. Martin, Algebraic convergence of discrete isometry groups of negative curvature, Pacific J. Math. 160 (1992) 109-127.

[McK] M.J.M. McKemie, Quasiconformal groups with small dilatation, Ann. Acad. Sci. Fenn. Ser. AI Math. 12 (1987) 95-118. MR 88j:30045

[Sul] D. Sullivan, On the ergodic theory at infinity of an arbitrary discrete group of hyperbolic motions, Riemann surfaces and related topics: proceedings of the 1978 Stony Brook Conference, Annals of Mathematics Studies 97, Princeton University Press, 1981, pp. 465-496. MR 83f:58052

[Tuk1] P. Tukia, On two dimensional quasiconformal groups, Ann. Acad. Sci. Fenn. 5 (1980) 73-78. MR 82c:30031

[Tuk2] P. Tukia, A quasiconformal group not isomorphic to a Möbius group, Ann. Acad. Sci. Fenn. Ser. AI Math. 10 (1985) 561-562.

[Tuk3] P. Tukia, Convergence groups and Gromov's metric hyperbolic spaces, New Zealand J. Math. 23 (1994) 157-187. MR 96c:30042

[Vai] J. Väisälä, Lectures on n-dimensional quasiconformal mappings, Springer-Verlag, Berlin, 1971. MR 56:12260

[Wat] P. Waterman, Möbius transformations in several dimensions, Adv. Math. 101 (1993) 87-113. MR 95h:30056

Department of Mathematics, The University of Michigan, Ann Arbor, Michigan 48109-1109

E-mail address: bonfert@math.lsa.umich.edu

Current address: Department of Mathematics, Wesleyan University, Middletown, Connecticut 06459

E-mail address: pbonfert@wesleyan.edu

Department of Mathematics, University of Auckland, New Zealand

E-mail address: martin@math.auckland.ac.nz 